\title{
DISEÑO E IMPLEMENTACIÓN DE UNA PLATAFORMA EXPERIMENTAL DE DOS GRADOS DE LIBERTAD CONTROLADA POR DOS TÉCNICAS: PID Y LÓGICA DIFUSA
}

\section{DESIGN AND IMPLEMENTATION OF AN EXPERIMENTAL PLATFORM WITH TWO DEGREES OF FREEDOM CONTROLLED BY TWO TECHNIQUES: PID \& FUZZY LOGIC}

\author{
Leonardo Solaque Guzmán', Cristhian Andrey Cristancho Cardozo², Camilo Andrés Gil Cárdenas ${ }^{3}$
}

Fecha de recepción: 30 de Enero de 2013

Fecha de aprobación: 21 de abril de 2014

Referencia: L. Solaque Guzmán, C.A. Cristancho Cardozo, C.A. Gil Cárdenas. (2014). Diseño e implementación de una plataforma experimental de dos grados de libertad controlada por dos técnicas: PID y Lógica Difusa. Ciencia e Ingeniería Neogranadina, 24 (1), pp. 99 - 115.

\section{RESUMEN}

El presente artículo tiene por objeto mostrar una estructura de dos grados de libertad, concebida y diseñada para aproximarse a la experimentación de leyes de control de sistemas tipo helicópteros de cuatro hélices (Quad-Rotor - Unmanned Aerial Vehicle). Se realizó el modelado y control para dicha plataforma. Dos tipos de control se abarcaron: uno basado en técnicas lineales tipo PID, que hace uso de la linealización por series de Taylor; y el otro basado en técnicas de control difuso. Se deja una plataforma abierta y, mediante la incursión en software-hardware in loop, se habilita para experimentar otras teorías de control.

Palabras clave: sistema de dos grados de libertad, sistemas aéreos no tripulados, linealización por series de Taylor, control PID, control difuso

\section{ABSTRACT}

This article is intended to show a structure of two degrees of freedom and two techniques of control. This system was conceived and designed in order to test different system control strategies able to command four-propeller-like helicopters (Quad-Rotor - Unmanned Aerial

\footnotetext{
1. IE, Ph.D., leonardo.solaque@unimilitar.edu.co, Profesor asociado, Facultad de Ingeniería, Investigador grupo GIDAM, Universidad Militar Nueva Granada, Bogotá, Colombia.

2. Ing. en Mecatrónica, cristhiancho8822@hotmail.com, Universidad Militar Nueva Granada, Bogotá, Colombia.

3. Ing. en Mecatrónica, camilocolombia@hotmail.com, Universidad Militar Nueva Granada, Bogotá, Colombia.
} 
Vehicle). This platform was modeled and controlled. Two control techniques were included: a linear technique PID-like control that makes use of the Taylor series linearization, and another technique based on fuzzy control. This work leaves an open platform and, through the software-hardware in loop approach, this platform is enabled to develop and test of other control theories.

Keywords: system with two degrees of freedom, unmanned aerial systems, Taylor series linearization, PID control, fuzzy control.

\section{INTRODUCCIÓN}

La Universidad Militar Nueva Granada dentro del marco de apoyo a la investigación en el programa de Ingeniería Mecatrónica grupo GIDAM, realiza una profundización en los temas que abarca la robótica aérea. El plantear el desarrollo de una plataforma de dos grados de libertad tipo helicóptero que simule los movimientos de cabeceo y guiñado, permite habilitar un prototipo para pruebas de estrategias de control. El objetivo es acercarse al diseño y control de sistemas más complejos, que se orienten a realizar vuelos autónomos, tal como se presenta en los vehículos aéreos no tripulados (Unmanned Aerial Vehicle) [1].

En general, el tema de los UAV viene tomando importancia desde hace ya más de una década. Quanser presenta plantas de dos grados de libertad como soporte en la enseñanza [2]. Humusoft presenta una plataforma similar también [3]. En [4], los autores presentan el control de vuelo estacionario de un helicóptero miniatura mediante el uso de identificación y técnicas multi-lazo. Un control GPC para la estabilización en pitch y yaw es presentado en [5]. Diferentes modos de vuelo con el fin de tener controles para estabilización son planteados en [6]. Técnicas de control difuso para el control de un Quad-Rotor se encuentran en el trabajo de maestría de Morata Palacios [7]. Un trabajo de modelado e identificación de parámetros puede ser visto en [8]. Una plataforma didáctica de dos grados es presentada en [9].

Bajo el contexto anterior, se pretende con este documento mostrar el diseño conceptual y la construcción de la plataforma de dos grados de libertad, y explicar las condiciones que debe cumplir, las características principales de la misma y los componentes mecánicos y electrónicos que la constituyen. Entre la instrumentación y motorización se encuentran los motores tipo brushless, controlados por drivers que regulan la velocidad por medio de una señal PWM; hélices acopladas a estos para la generación del empuje y lograr movimiento de cabeceo (pitch) y de guiñada (yaw); central inercial referencia 0S-4000 que genera una trama de datos con los valores de orientación de la plataforma; una tarjeta de adquisición para recopilar los datos del sensor y la generación de señales de control de los actuadores; y finalmente, 
una interfaz visual en un computador que comunica serialmente a la tarjeta antes mencionada. Se presenta el desarrollo de los controles, en el que se hace el modelado de la plataforma de dos grados de libertad por medio de la técnica "Newton-Euler". La linealización por "Taylor" es usada de manera que la teoría de control lineal se aplique. Posteriormente se explican los controladores desarrollados, basados en técnicas de control clásico como el PID y control moderno como la lógica difusa. Se utilizan técnicas de simulación basadas en la herramienta de MATLAB- Simulink para obtener el comportamiento del modelo y hacer la validación de los controladores para el mismo. Resultados de la implementación sobre la plataforma real son presentados al final del documento, al igual que las conclusiones.

\section{DISEÑO CONCEPTUAL Y CONSTRUCCIÓN}

La plataforma experimental tipo helicóptero tiene dos grados de libertad, que permiten rotar libremente alrededor del eje y conocido como pitch y alrededor del eje z - conocido como yaw, y habilitan la posibilidad de aplicar técnicas de control como reguladores difusos, lineales y no lineales, entre otros.

Para la elaboración de la plataforma se tuvo en cuenta que fuera una estructura rígida y fuerte, capaz de soportar las fuerzas propias del sistema, lograr reconfiguración mediante la ubicación de sus rotores, tal que permita cumplir configuraciones de tipo Tail rotor(Movimiento alrededor del eje Y. Cabeceo), y Tandem rotor (Movimiento alrededor del eje Z. Alabeo). La revisión del estado del arte, evidenció la proyección de una plataforma que permita facilidades de construcción y ensamblaje. El prototipo se diseñó en el software de modelado 3D SolidWorks y se describe en la Figura 1.

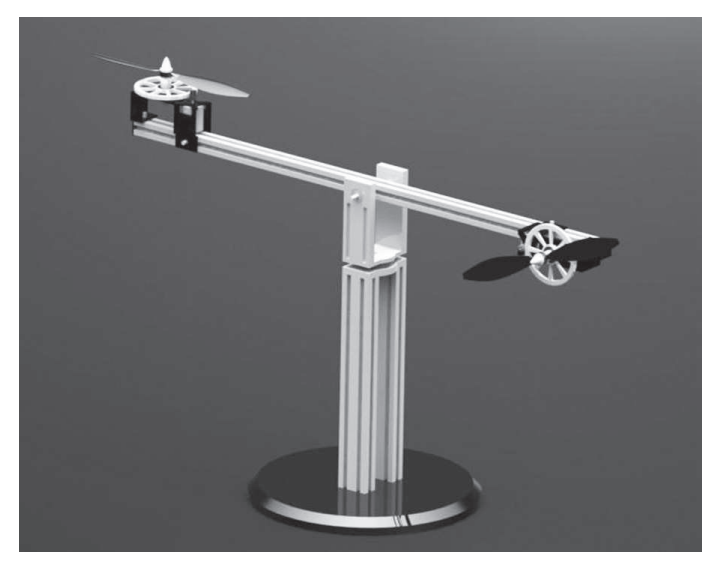

Figura 1: Diseño 3D Plataforma de dos grados de libertad, SolidWorks. 
Acontinuaciónsedescribenloscomponentes que constituyen la plataforma:

\subsection{MOTORES}

Loscomponentesprincipalesdelaplataforma son los motores, los cuales se encargan de generar las fuerzas de empuje que permiten producir movimiento en los dos grados de libertad. Los motores escogidos para esta aplicación fueron dos Turnigy X500-4000 que alcanzan una velocidad máxima de 34000 rpm y son comúnmente utilizados en helicópteros de aeromodelismo, lo que permite tener una aproximación real a los elementos que puedan ser utilizados en el diseño de un UAV tipo helicóptero. Estos cuentan con una etapa de reducción que permite obtener una menor velocidad y un mayor torque inducido a las hélices, proporcionando el acople de distintos tamaños de ellas y la experimentación de efectos aerodinámicos producidos.

\subsection{SENSOR}

Parte importante en un sistema de control es el sensor. Éste permite la realimentación de los estados de la planta, que para este caso son los ángulos de cabeceo y guiñada (pitch y yaw, respectivamente). Para cumplir esta tarea se utilizó un Compass OS-4000 distribuido por la empresa Ocean Server. Este sensor posee acelerómetros en el eje $x$, $y, z, y$ genera las señales de los tres ángulos de orientación, pitch, yaw y roll. El sensor transmite esta información mediante un protocolo de comunicación Serial - TTL y a una velocidad máxima de 119000 bps.

\subsection{TARJETA DE ADQUISICIÓN DE DATOS}

Para la adquisición de datos y generación de las señales de control a los motores se diseñó una tarjeta con un microcontrolador 18F4550, que permite la recepción de datos del sensor por medio de comunicación serial, conexión USB 2.0 para enviar y recibir datos del y al computador, y generación de señales de PWM para el control de los drivers. El esquema general de la tarjeta se describe en la Figura 2.

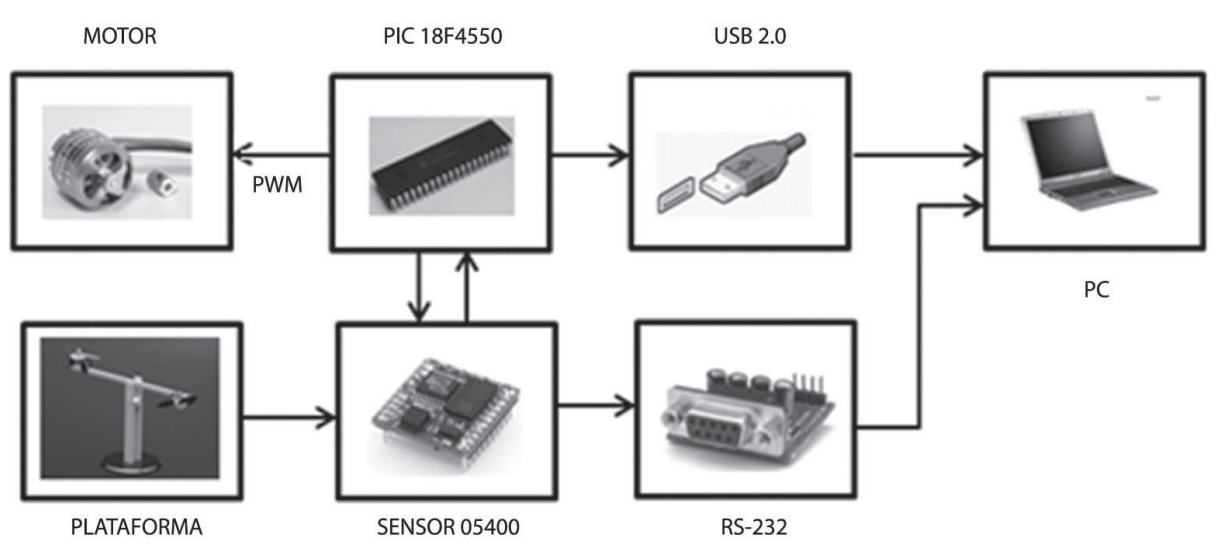

Figura 2: Diseño conceptual de tarjeta de control y adquisición de datos. 


\subsection{INTERFAZ}

Se implementó una interfaz de software amigable con el usuario. El objetivo se centra en poder brindar al usuario la posibilidad de integrar nuevos controles o probar los desarrollados por las técnicas de PID o lógica difusa. La Figura 12 ilustra esta interfaz de usuario.

Cabe resaltar que, aun cuando la interfaz de usuario no tiene como propósito poder hacer el diseño de los controles, sí permite integrar archivos con entrada-salida específicas. Esto es, sabiendo qué variable se quiere comparar con la referencia que se ingresa por usuario, se toma de las variables adquiridas por el sistema micro-controlador (sujetas a al protocolo de comunicación) y se integra al archivo que contenga el control en ecuaciones en diferencia - PID o Lookup table - difuso. De igual forma, se asocia la variable de salida de dicho archivo, a la variable que se quiere controlar (potencia de los motores).

\subsection{CONSTRUCCIÓN}

La Figura 3 presenta la plataforma construida y la tarjeta de adquisición y procesamiento de datos que se utilizó para la implementación de los controladores.
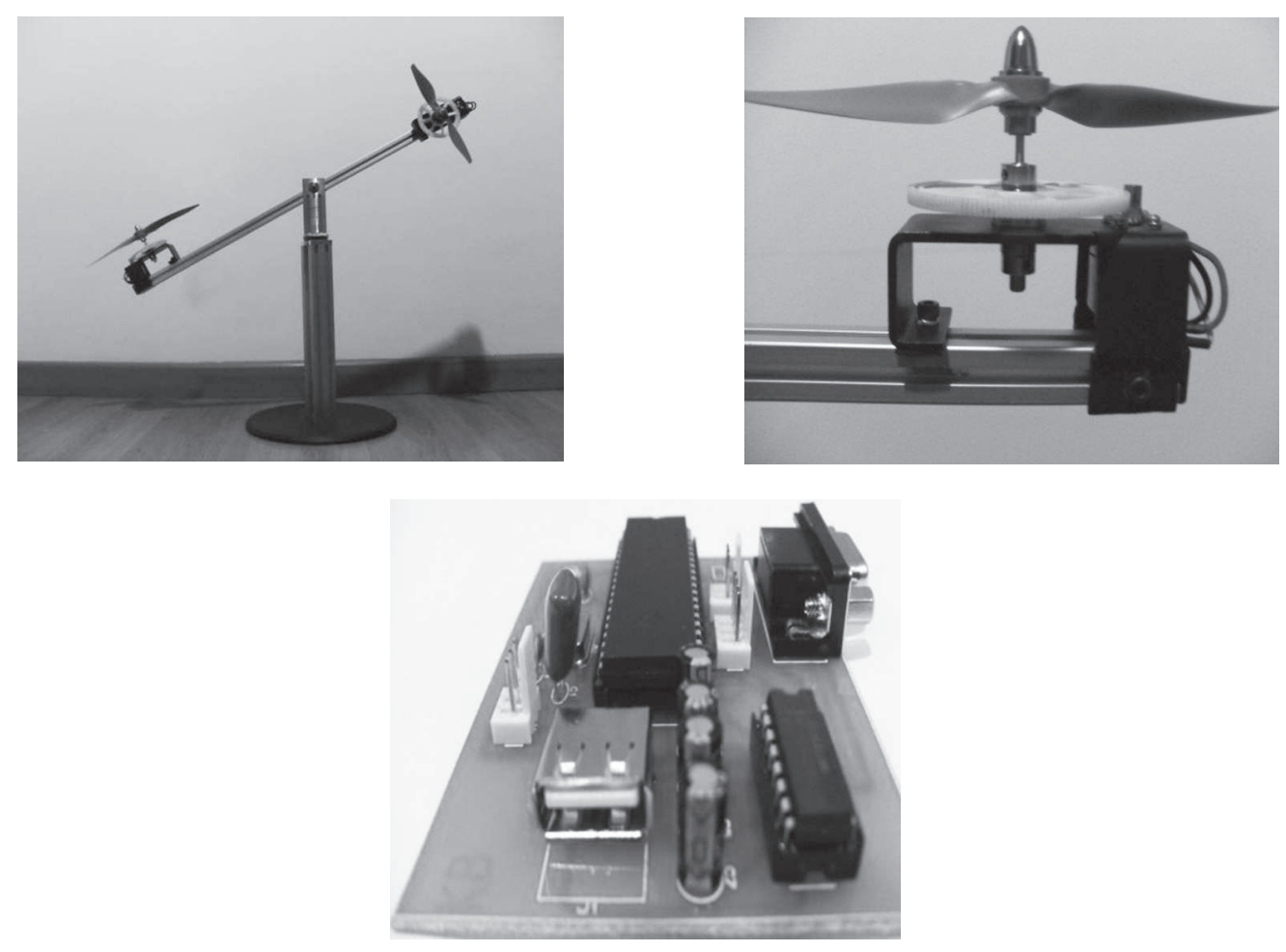

Figura 3: Imágenes de la plataforma y tarjeta construidas. 


\section{MODELO MATEMÁTICO}

Un helicóptero es un sistema complejo de modelar debido a los efectos dinámicos, aerodinámicos y mecanismos que lo componen y que intervienen en su movimiento. Para el desarrollo del modelo de un helicóptero tipo aeromodelo, y en especial para el modelo de dos grados de libertad que aquí se propone, es necesario hacer las siguientes hipótesis:

- La estructura se supone como estructura rígida.

- El sistema se considera estacionario, es decir que no posee desplazamiento en ninguno de los ejes coordenados.
- Las hélices son consideradas como cuerpos rígidos.

- Las fuerzas de empuje y los torques generados por los motores - hélices son directamente proporcionales al cuadrado de la velocidad angular.

Para cualquier sistema mecánico se puede desarrollar un modelo matemático representativo de su dinámica, aplicando las leyes de Newton. En la Figura 4 se puede observar el diagrama de cuerpo libre del sistema y las fuerzas que actúan sobre él.

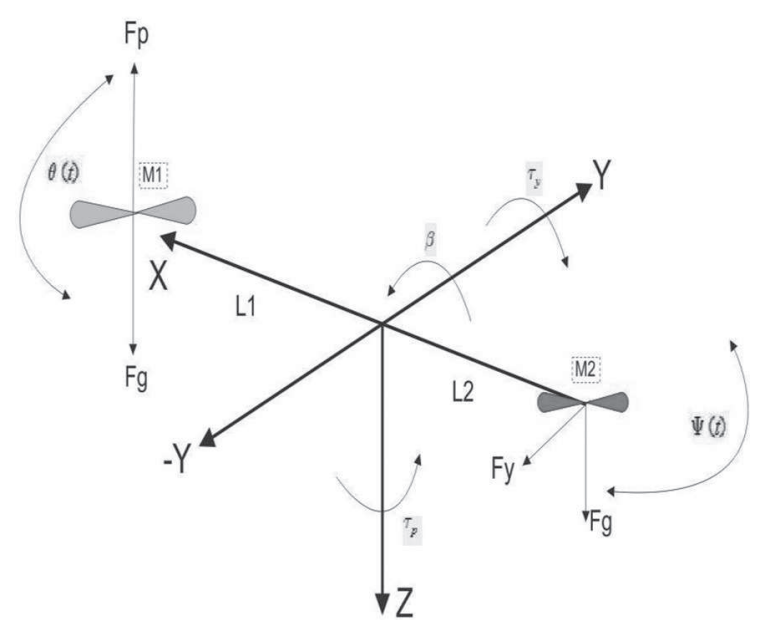

Figura 4: Diagrama de cuerpo libre de la configuración Tail rotor.

Aplicando la segunda ley de Newton sobre cada eje en el que se refleja movimiento, se tiene que:

$$
\begin{gathered}
\sum T=J_{p} \theta \\
J_{p} \ddot{\theta}(t)=F_{p}(t) L_{1}-\mu_{p} \dot{\theta}(t)-M_{1} g L_{1} \operatorname{Sen}(\theta(t))+M_{2} g L_{2} \operatorname{Sen}(\theta(t))+K_{t y} F_{y}(t)
\end{gathered}
$$




$$
\begin{gathered}
\sum_{T=J_{y} \ddot{\varphi}} \\
J_{y} \ddot{\varphi}(t)=F_{y}(t) L_{2}-\mu_{y} \ddot{\varphi}(t)+K_{t p} F_{p}(t)
\end{gathered}
$$

Donde:

- $J_{p} y J_{y}$ son los momentos de inercia del cuerpo alrededor de $\theta$ y $\varphi$, los cuales se calculan mediante las fórmulas de inercia rotacional a través de un punto, y la fórmula de inercia de una barra. Como se tienen dos concentraciones de masa en los extremos y una barra la inercia total será la suma de las tres inercias individuales.

$$
J_{\text {total }}=L_{1}{ }^{2} M_{1}+L_{2}{ }^{2} M_{2}+\frac{1}{3} M L^{2}
$$

- $\quad \theta *$ у $\varphi$ “ son las aceleraciones angulares de pitch y yaw respectivamente.

- $F_{p}$ y $F_{y}$ son las fuerzas de empuje que son proporcionales a las señales PWM que manejan los drivers de los motores. Para la determinación de esta relación, mediante una planta experimental, se hizo la caracterización de empuje generado en función del ancho de pulso aplicado. Esta relación se describe en la Figura 5.

- $K_{t p} y K_{t y}$ son las constantes de torque de reacción generadas por la acción de los motores.

- $\mu_{p}$ y $\mu_{y}$ son los coeficientes de fricción. Estas constantes son halladas por medio de la ecuación que proporciona el fabricante de los rodamientos SKF:

$$
\mu=0.5 u P d
$$

donde $\mathrm{u}$ es el coeficiente de fricción constante del rodamiento y viene especificado en la tabla de características, $P$ es la carga dinámica equivalente, y del diámetro interno del rodamiento.

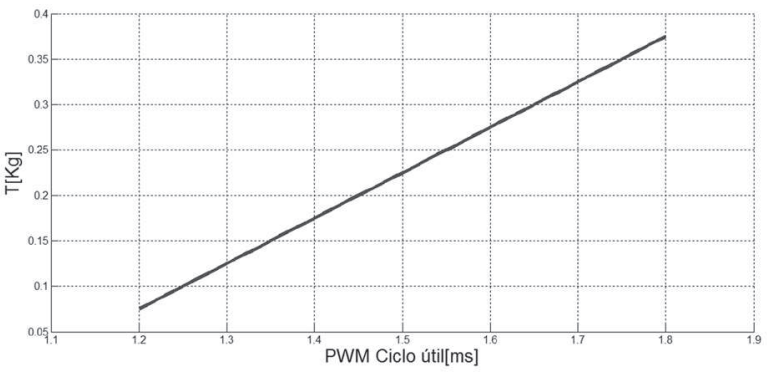

Figura 5: Empuje real de la hélice APC 10×6 en función del PWM.

- $M_{1}$ y $M_{2}$ son las masas concentradas en cada uno de los extremos.

- $L_{1}$ y $L_{2}$ son las distancias de los motores con respecto al centro de rotación

\subsection{REDUCCIÓN DE MODELOS}

Para obtener un modelo de un helicóptero de dos grados de libertad que permita la implementación de reguladores por técnicas lineales para su control es necesario desarrollar un modelo simplificado. Partiendo de las ecuaciones (1) y (2), se aplica la teoría de linealización por series de Taylor ([10-11] presentan profundización en el tema).

Para el movimiento de pitch, se asume no movimiento en yaw y que la fuerza de empuje 
$F_{y}=0$. Esto elimina posibles acoples entre las dinámicas de cada eje, $\varphi=\varphi^{*}=\ddot{\varphi}=0$. La ecuación (1) puede ser reescrita de la siguiente forma:

$$
\begin{gathered}
K_{1}=M_{1} g L_{1}-M_{2} g L_{2} \\
J_{p} \ddot{\theta}(t)=F_{p}(t) L_{1}-\mu_{p} \dot{\theta}(t)-K_{1} \sin (\theta(t))
\end{gathered}
$$

Debido a que la ecuación $\left(1^{*}\right)$ presenta un comportamiento no lineal, se acude a la teoría de linealización de Taylor para encontrar su equivalente linealizado y en variaciones. Así, asumiendo variaciones sobre las variables de estado del sistema (posiciones y velocidades angulares en pitch y yaw) se obtiene:

$$
\dot{\theta}(t)=\omega_{p}
$$

$\dot{\omega}_{p}(t)=\frac{1}{J_{p}}\left(F_{p}(t) L_{1}-\mu_{p} \dot{\theta}(t)-K_{1} \sin (\theta(t))\right)=f_{2}$

donde:

$$
\dot{\theta}(t)=\omega_{p}
$$

$\dot{\omega}_{p}(t)=\frac{\partial f_{1}}{\partial \theta}(\theta-\bar{\theta})+\frac{\partial f_{1}}{\partial \omega}(\omega-\bar{\omega})+\frac{\partial f_{1}}{\partial F_{p}}\left(F_{p}-\bar{F}_{p}\right)$

siendo

$\delta \theta=\theta-\bar{\theta} ;$ variación de pitch

$\delta \omega=\omega-\bar{\omega} \delta \theta=\theta-\bar{\theta} ;$ variación de la velocidad angular del ángulo pitch

$\delta F_{p}=F_{p}-\bar{F}_{p} \delta \theta=\theta-\bar{\theta} ;$ variación propulsión del motor M1.

La expresión linealizada sobre el punto donde operará puede ser escrita como:

$$
J_{p} \delta \ddot{\theta}(t)=\delta F_{p}(t) L_{1}-\mu_{p} \delta \dot{\theta}(t)-K_{1} \delta \theta(t)
$$

Teniendo la ecuación linealizada, se halla la función de transferencia que será usada para el diseño de los reguladores. Para esto se aplica la transformada de Laplace $\theta(\mathrm{s})=\mathrm{L}(\theta)$.

$$
\begin{gathered}
J_{p} \delta \ddot{\theta}(t)+\mu_{p} \delta \dot{\theta}(t)-K_{1} \delta \theta(t)=\delta F_{p}(t) L_{1} \\
\delta \theta(S)\left[J_{p} S^{2}+\mu_{p} S+K_{1}\right]=\delta F_{p}(S) L_{1} \\
\frac{\delta \theta(S)}{\delta F_{p}(S)}=\frac{\frac{L_{1}}{J_{p}}}{S^{2}+\frac{\mu_{p}}{J_{p}} S+\frac{K 1}{J_{p}}}
\end{gathered}
$$

Ahora, para el movimiento que rige el comportamiento dinámico del yaw, asumiendo hipótesis similares al anterior caso, $\theta=\theta^{\circ}=\theta^{*}=0, F_{-}$p $=0$ y la ecuación (2) se tiene:

$$
J_{y} \ddot{\varphi}(t)=F_{y}(t) L_{2}-\mu_{y} \dot{\varphi}(t)
$$

Como se observa, la ecuación $\left(2^{*}\right)$ tiene un comportamiento lineal. La excepción es el acople dinámico que existe con pitch, pero por la hipótesis se asumirá no movimiento en ese eje. Aplicando la transformada de Laplace,

$$
J_{y} \ddot{\varphi}(t)+\mu_{y} \dot{\varphi}(t)=F_{y}(t) L_{2}
$$

$$
\varphi(S)\left[J_{y} S^{2}+\mu_{y} S\right]=F_{y}(S) L_{2}
$$

se obtiene

$$
\frac{\varphi(S)}{F_{y}(S)}=\frac{\frac{L_{2}}{J_{y}}}{S^{2}+\mu_{y} S}
$$




\subsection{SIMULACIÓN DE MODELOS}

Las simulaciones de los modelos completos se realizaron mediante la herramienta Simulink. En la Figura 6 se describe el

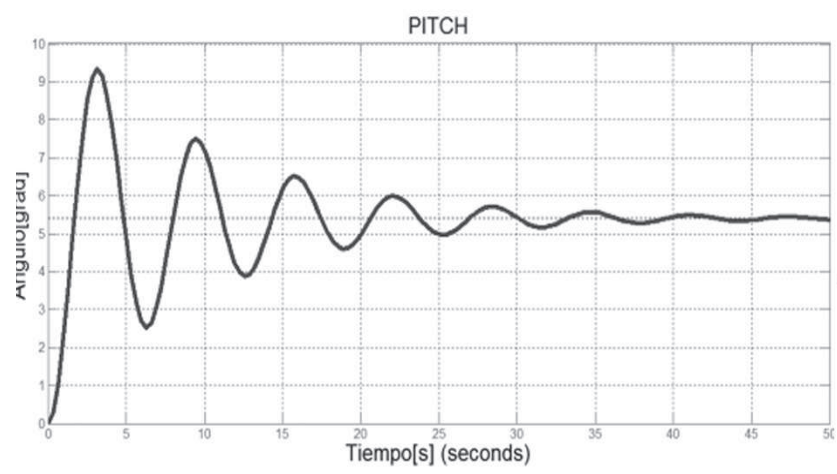

comportamiento para los ángulos de pitch y yaw ante una entrada escalón unitario.

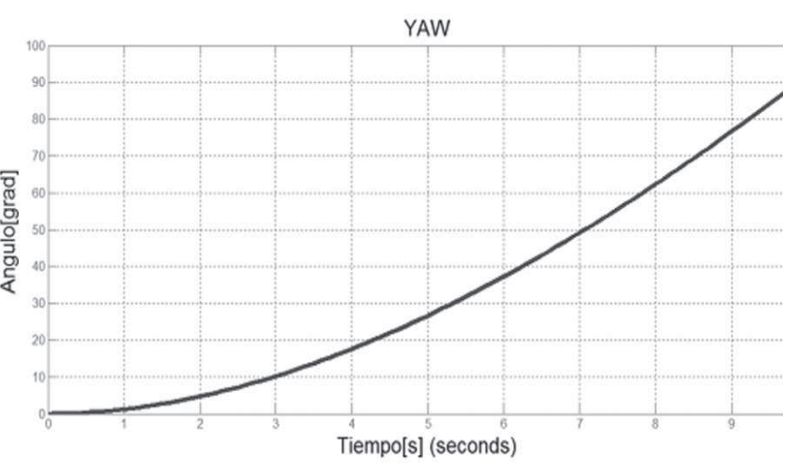

Figura 6: Respuesta del modelo no lineal en simulación.

Se observa que debido a la dinámica del sistema y a las restricciones mecánicas de la plataforma, el ángulo de pitch presenta oscilaciones decrecientes. A diferencia del ángulo de pitch, el comportamiento del yaw es una respuesta exponencial creciente o decreciente en el tiempo ya que no existe una restricción que estabilice el sistema.
Una prueba sobre la estructura presenta comportamientos similares, validando los tiempos observados en la anterior gráfica.

EnlaFigura 7 sedescribeel comportamiento del modelo lineal para los ángulos de pitch y yaw.
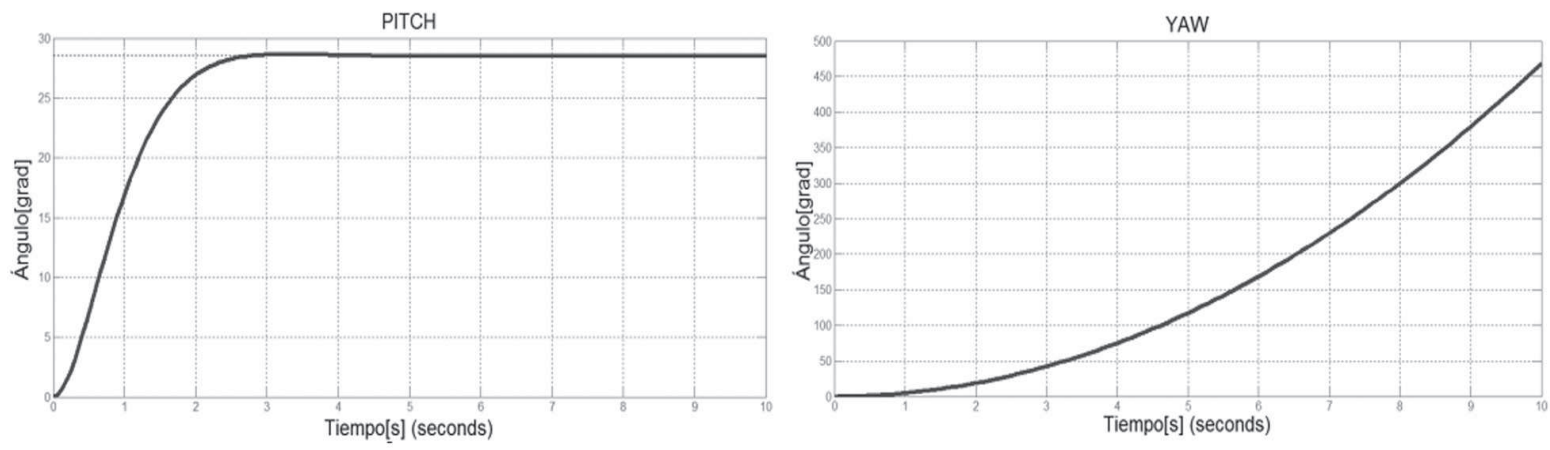

Figura 7: Respuesta del modelo lineal en simulación. 
Las simulaciones del modelo lineal se hicieron con una entrada escalón de 0,1, cercana al punto de operación 0 , el cual fue establecido en la liberación del brazo. Se observa cómo la respuesta de los modelos es más rápida y sin oscilaciones. Este es un comportamiento esperado en un sistema linealizado. Así, están los modelos para el diseño de los controles lineales.

\section{DISEÑO DE CONTROLADORES PID}

El cálculo del regulador de PID implica tres acciones de control diferentes: la acción proporcional, la integral y la derivativa. El valor proporcional determina la acción al error actual, el integral determina la reacción basada en la suma de errores recientes y el derivado determina la anticipación a cambios en el error. La suma de estas tres acciones se utiliza para ajustar el proceso de control de un sistema. Para este caso, la planta se establece como un sistema SISO (single input, single output), por lo cual se hizo el diseño de un regulador PID para cada ángulo.

Para los parámetros de diseño se tiene como partida el desempeño del sistema en lazo abierto. Generalmente recaen como sistemas de doble integrador. Desde la teoría, estos sistemas tienden a infinito cuando tienen por entrada un escalón. Para el caso, asumiendo la reacción propia del sistema (fuerza de empuje de motores + hélice e inercias vencidas), se asumen tiempos de estabilización no superiores a los 5 segundos, con un máximo sobrepaso del $25 \%$. Con estos requerimientos, se procedió a realizar el cálculo de los reguladores.
Partiendo del modelo descrito por la ecuación (3).

$$
\frac{\delta \theta(S)}{\delta F_{p}(S)}=\frac{\frac{L_{1}}{J_{p}}}{S^{2}+\frac{\mu_{p}}{J_{p}} S+\frac{K_{i}}{J_{p}}}
$$

se diseña un regulador PID con la siguiente función de transferencia:

$$
G_{c}(S)=K_{p}+K_{d} S+\frac{K_{i}}{S}
$$

Por la técnica de asignación de polos, se obtiene la función de transferencia en lazo cerrado.

$$
G_{C}(S)=\frac{\frac{L_{1}}{J_{p}} K_{d} S^{2}+\frac{L_{1}}{J_{p}} K_{p} S+K_{i}}{S^{3}+\frac{\mu_{p}}{J_{p}} S^{2}+\frac{K_{i}}{J_{p}} S+\frac{L_{1}}{J_{p}} K_{d} S^{2}+\frac{L_{1}}{J_{p}} K_{p} S+K_{i}}
$$

obteniendo el polinomio característico

$$
B(S)=S^{3}+\left(\frac{\mu_{p}}{J_{p}}+\frac{L_{1}}{J_{p}} K_{d}\right) S^{2}+\left(\frac{K_{i}}{J_{p}}+\frac{L_{1}}{J_{p}} K_{p}\right) S+K_{i}
$$

Con el polinomio deseado de la forma $B_{d}$ $(S)=\left(S^{2}+2 \zeta W n S+W^{2}\right)(S+\alpha)$, sujeto a los criterios de desempeño deseados en lazo cerrado, se igualan los términos de los polinomios y se determinan las ganancias del controlador:

$$
\begin{aligned}
S^{3} & =S^{3} \\
2 W n S^{2}+\alpha S^{2} & =\left(\frac{\mu_{p}}{J_{p}}+\frac{L_{1}}{J_{p}} K_{d}\right) S^{2} \\
2 \rho W n+\alpha & =\left(\frac{\mu_{p}}{J_{p}}+\frac{L_{1}}{J_{p}} K_{d}\right) \\
2 \rho W n \alpha+W n^{2} & =\left(\frac{K_{i}}{J_{p}}+\frac{L_{1}}{J_{p}} K_{p}\right) \\
\alpha W n^{2} & =\frac{L_{1}}{J_{p}} K_{i}
\end{aligned}
$$


de donde, según los criterios de diseño antes planteados, se encuentran, por la técnica de asignación de polos, los coeficientes del regulador: $\mathrm{Kp}=73.86, \mathrm{Ki}=$ 103.03 y $\mathrm{Kd}=25.89$.

Para el diseño del regulador para el movimiento alrededor del eje ' $Z$ ' lángulo de yaw) se tomó como base la función de transferencia encontrada para el modelo reducido descrito por la ecuación (4).

$$
\frac{\varphi(s)}{F_{y}(s)} \frac{\frac{L_{2}}{J_{y}}}{S^{2}+\mu_{y} S}
$$

Al igual que el diseño del regulador de pitch, se utilizó un procedimiento similar que encontró las constantes del regulador $K_{p}=321.5, K_{i}=454.44, K_{d}=110.32$.

\subsection{ANTI-WIND-UP}

Generalmente la acción integral actúa siempre que hay error, así la señal en el actuador haya llegado a su máximo, es decir a su saturación. Una técnica que ayuda a mitigar la acción integral en este caso es la denominada anti-wind-up. Ésta inhibe la acción integral cuando el error perdura y el actuador se encuentra en sus valores máximos (la señal de control

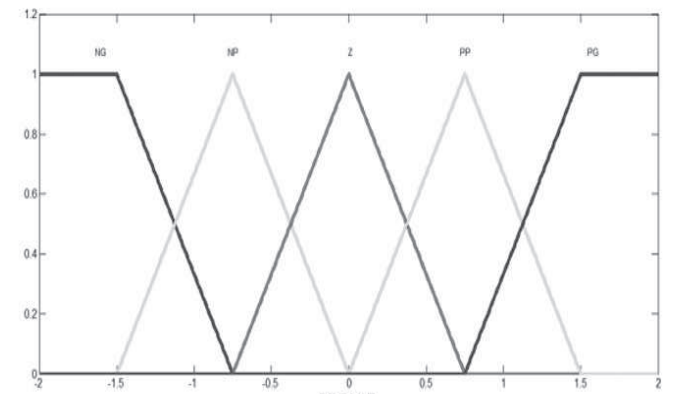

puede irse $\mathrm{a}+/$ - infinito y puede conducir inestabilidades en el sistema). Pruebas en simulación fueron realizadas de manera que se validara la estabilidad del sistema. Luego se hizo la integración al montaje real presentando resultados apropiados.

\section{DISEÑO DE CONTROLADORES DIFUSOS}

La lógica difusa es una rama de la inteligencia artificial que permite analizar información del mundo real en una escala entre falso y verdadero. La lógica difusa puede controlar o describir un sistema usando reglas de sentido común (conocimiento de experto) que se asocian a variables físicas ([12] presenta una profundización en el tema).

Para el diseño del regulador por técnicas de lógica difusa, se utilizó un control difuso PD. Este utiliza el error e(k) y el cambio de error de(k)/dt para producir cambios en la función de salida del controlador (puede ser $u(k)$ y du(k)) .

Para el error y la derivada del error (ver Figura 8) se definieron las variables negativamente grande (NG), negativamente pequeño (NP), aproximadamente cero (Z), positivo pequeño (PP) y positivo grande (PG).

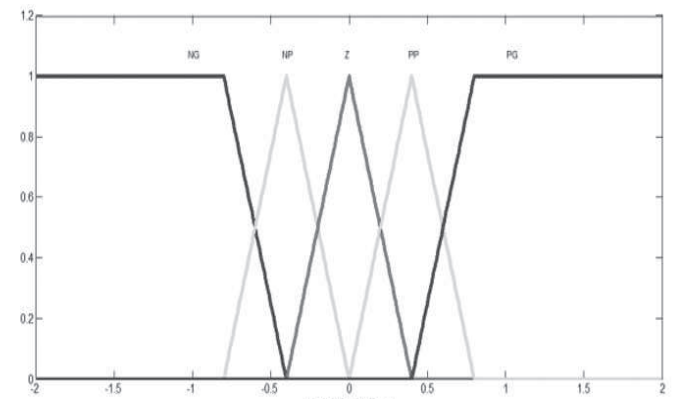

Figura 8: Conjuntos borrosos para el error y la derivada del error. 
De igual forma se definieron los conjuntos pequeño (NP), aproximadamente cero (Z), borrosos de la salida (ver Figura 9): positivo pequeño (PP) y positivo grande (PG). negativamente grande (NG), negativamente

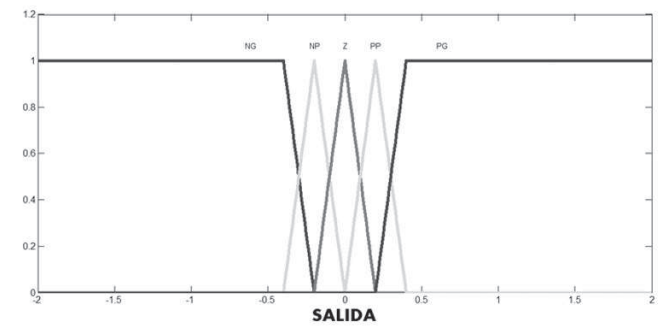

Figura 9: Conjuntos borrosos para la salida.

Utilizando el conocimiento que se tiene del controlador y están directamente acerca de la planta se desarrollaron las relacionadas con las variables lingüísticas, reglas del controlador difuso. Se plantearon tal como se muestra en la Tabla 1

25 reglas que representan la cuantificación

Tabla 1: Reglas de inferencia

\begin{tabular}{|c|c|c|c|c|c|}
\hline$\frac{d}{d t} e(t) \backslash e(t)$ & $\mathrm{Ng}$ & $\mathrm{Np}$ & $\mathrm{Z}$ & $\mathrm{Pp}$ & $\mathrm{Pg}$ \\
\hline $\mathrm{Ng}$ & $\mathrm{Ng}$ & $\mathrm{Np}$ & $\mathrm{Np}$ & $\mathrm{Np}$ & $\mathrm{Np}$ \\
\hline $\mathrm{Np}$ & $\mathrm{Np}$ & $\mathrm{Z}$ & $\mathrm{Pp}$ & $\mathrm{Np}$ & $\mathrm{Z}$ \\
\hline $\mathrm{Z}$ & $\mathrm{Np}$ & $\mathrm{Pp}$ & $\mathrm{Z}$ & $\mathrm{Pp}$ & $\mathrm{Pp}$ \\
\hline $\mathrm{Pp}$ & $\mathrm{Z}$ & $\mathrm{Pp}$ & $\mathrm{Pp}$ & $\mathrm{Z}$ & $\mathrm{Pp}$ \\
\hline $\mathrm{Pg}$ & $\mathrm{Pp}$ & $\mathrm{Pp}$ & $\mathrm{Pp}$ & $\mathrm{Pp}$ & $\mathrm{Pg}$ \\
\hline
\end{tabular}

El paso seguido dentro de la lógica difusa es la defusificación, la cual va a dar la salida del controlador lógico difuso. El método usado para encontrar la salida del controlador fue el algoritmo Root Sum Squared - RSS (centroide), dado por la ecuación (5). Este permitió encontrar un valor asociado a los conjuntos difusos.

$$
\text { Centroide }=\frac{\int f(x) x d x}{\int f(x) d x}
$$

\section{RESULTADOS Y ANÁLISIS}

Se pretende en esta sección, mostrar los resultados obtenidos en las pruebas a nivel de simulación como etapa previa a la implementación. Resultados experimentales también se presentan a continuación.

\subsection{PRUEBAS EN SIMULACIÓN}

Las simulaciones de los reguladores PID de posición para el ángulo de pitch y yaw se desarrollaron mediante la herramienta 
de software MATLAB/Simulink. La Figura 10 ilustra los resultados obtenidos con los reguladores PID. En ella se observa que los reguladores responden apropiadamente, con tiempos de estabilización de 3 a 4 segundos. Los sobreimpulsos, aunque no son deseados, permiten llegar a una referencia deseada de manera rápida.
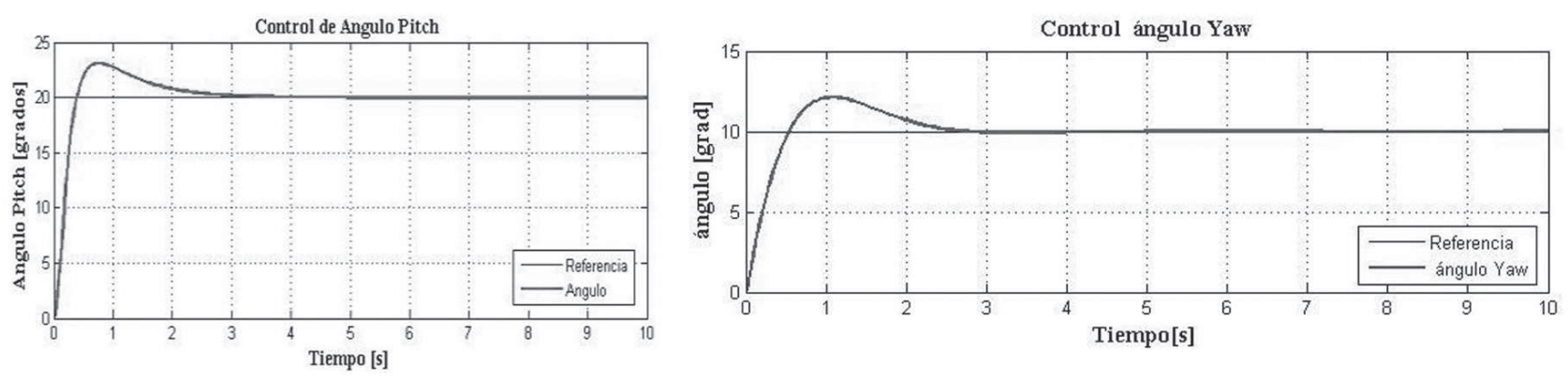

Figura 10: Control PID de los ángulos de Pitch y Yaw.

La Figura 11 ilustra el comportamiento de los reguladores difusos. En ella se puede observar que presentan una mayor oscilación antes de alcanzar su régimen permanente, es decir su estabilidad. Al igual que el anterior desempeño, se encuentra que se estabilizan sobre los 3 a 4 segundos.
De manera general, se puede decir que se tienen comportamientos deseables, según los criterios de diseño de los controles en el ámbito de simulación.
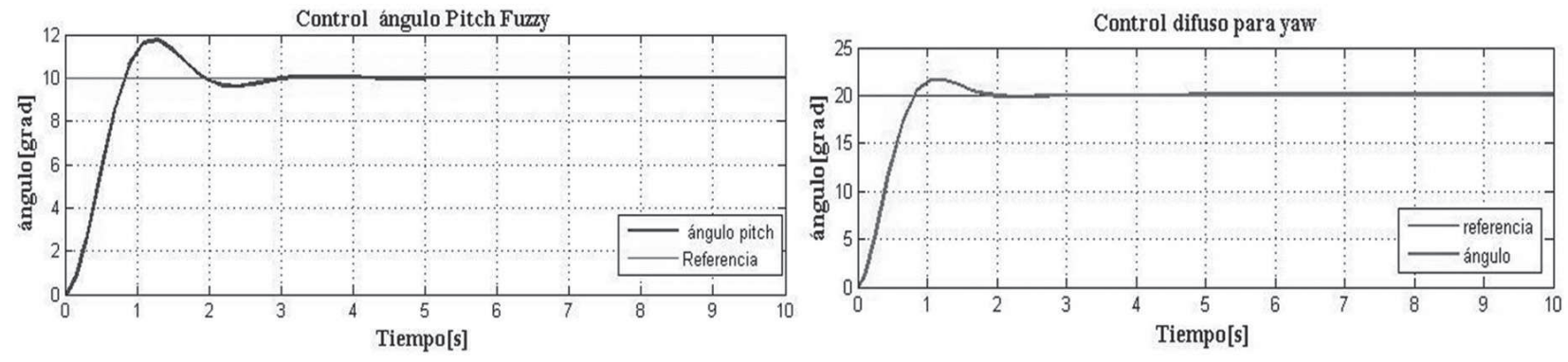

Figura 11: Control difuso para los ángulos de Pitch y Yaw. 


\subsection{PRUEBAS EXPERIMENTALES}

Para realizar pruebas experimentales de los controladores diseñados sobre la plataforma real. Se utilizaron dos técnicas:

a. Aproximación a "Software - In the - Loop". Consiste en hacer la programación de los controladores y decodificar las señales del sensor dentro de un computador y utilizar el hardware o microprocesador 18 F4550 para la transmisión de datos entre la planta real y el software de control. La implementación de los controladores se hizo en lenguaje C\# y la transmisión de datos que se utilizó fue USB 2.0 para el procesamiento de datos y RS-232 para la recepción de las señales del sensor. b. Aproximación a "Hardware-Inthe-Loop". Se basa en programar los controladores dentro del hardware, que para este caso es el microcontrolador 18F4550. En éste se realizan todos los cálculos con la señal de referencia que le llega del computador y la señal de realimentaciónquellega directamente al PIC. El lenguaje utilizado es C y es implementado mediante el software PIC C. Para este caso el computador solo se encarga de la visualización de variables y la reproducción de los movimientos de la plataforma en tiempo real. La Figura 12 ilustra esto.

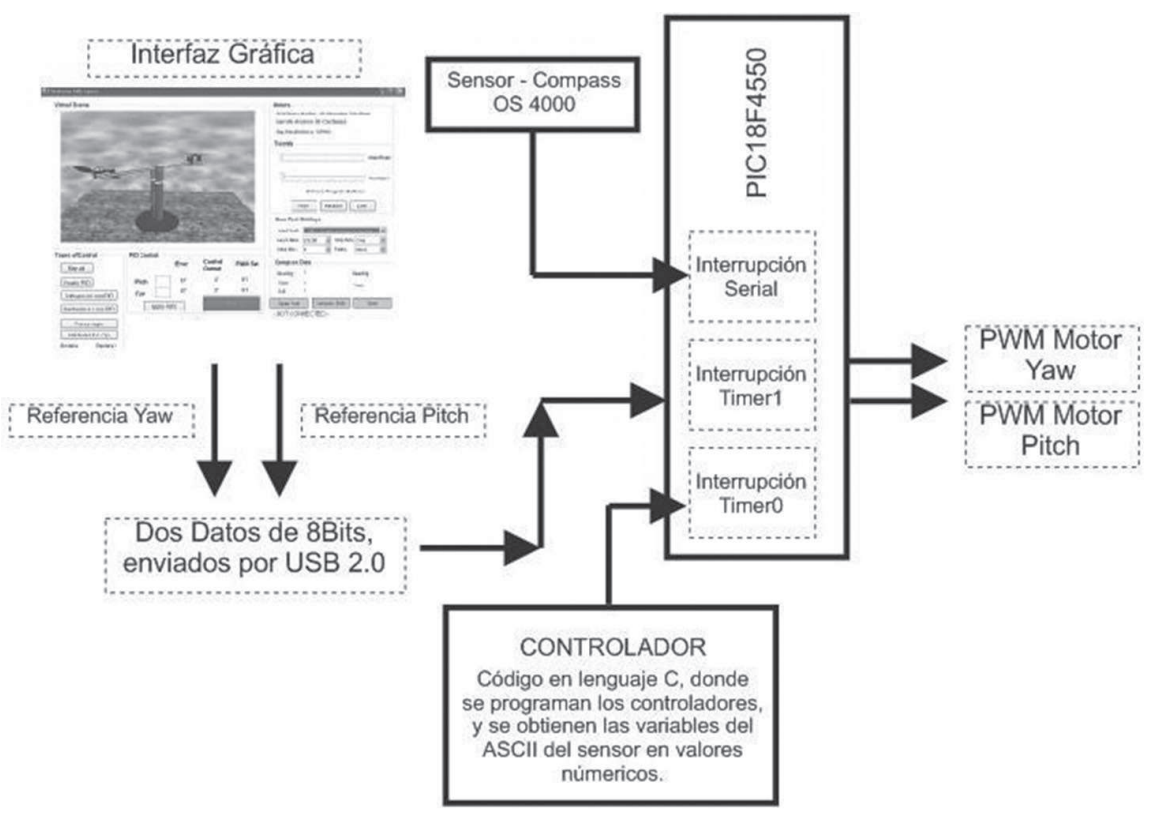

Figura 12: Diagrama de "Hardware in the loop". 
En las primeras pruebas que se realizaron con el control PID en la estructura real, se observó que había variaciones bruscas y la respuesta tardaba en estabilizarse. Por consiguiente se procedió a hacer una sintonización fina, que logró ajustar a respuestas similares a las obtenidas en simulación. Como se observa en la Figura 13, el sistema presenta pequeños sobrepicos lo cual hace la respuesta de la planta más lenta, pero siempre en busca de su señal de referencia.
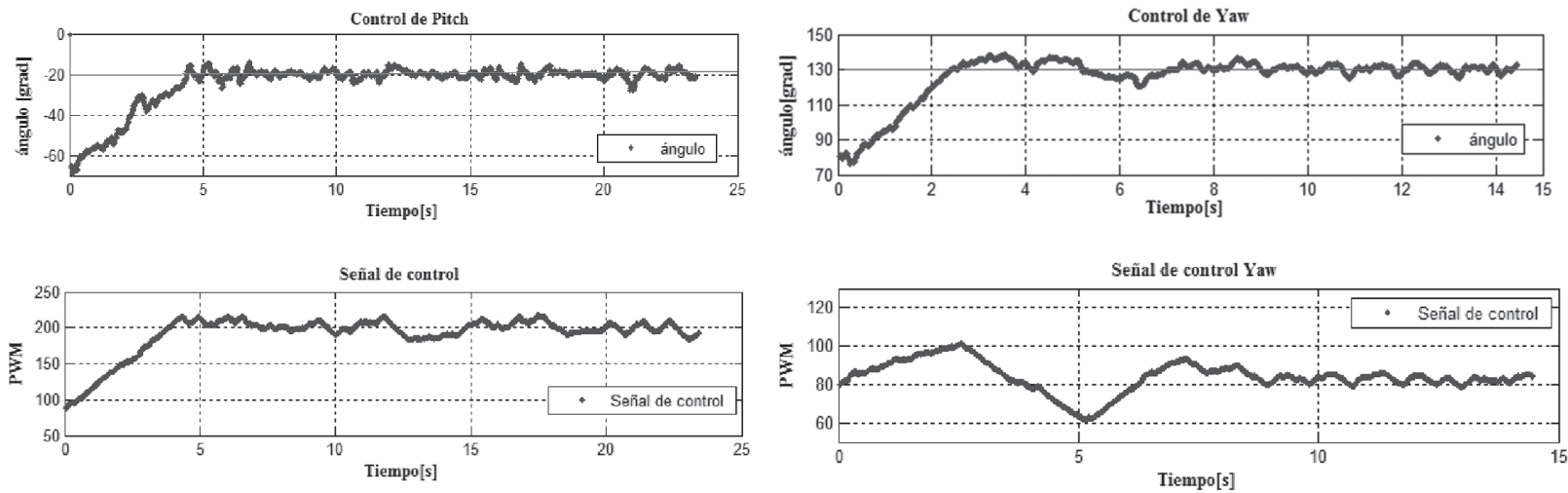

Figura 13: Control PID para los ángulos de pitch y yaw

La Figura 14 muestra los resultados del control difuso para el ángulo pitch. Se observa que la salida del sistema converge a la referencia, logrando establecerse alrededor de 8 segundos. Para el
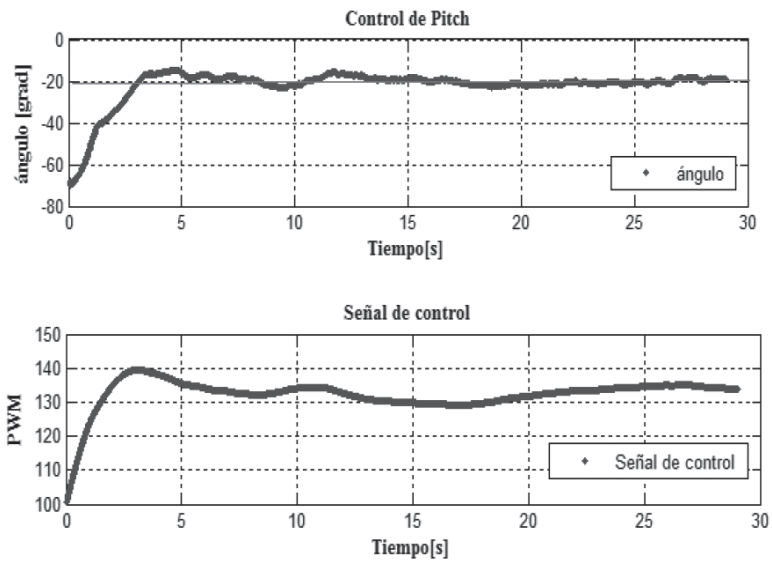

ángulo de yaw, la respuesta tiene un comportamiento más sub-amortiguado, y mantiene tiempos casi similares a los del pitch en cuanto a la estabilización.
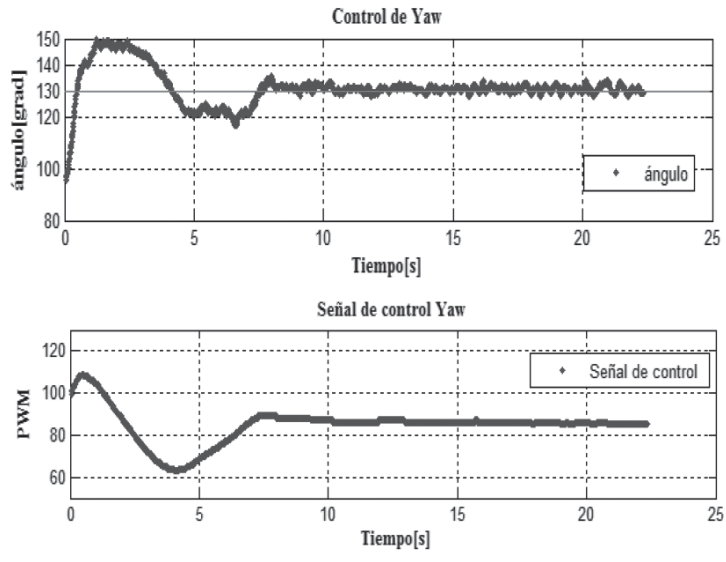

Figura 14: Control difuso para los ángulos de pitch y yaw. 


\section{CONCLUSIONES}

Basados en el principio de concebir una estructura con dos grados de libertad, que se asemejara a un sistema aéreo de rotores fijos tal como los Quad-Rotor, se diseñó y construyó una plataforma que permitiera hacer aproximación al modelado de ella y su control. Así, se realizó el modelado de la estructura concebida, tipo helicóptero de dos grados de libertad, con base en la teoría de Newton-Euler.

La teoría de linealización por Taylor permitió, a partir del modelo matemático encontrado, realizar lareducción del modelo complejo (no lineal), bajo la hipótesis de condiciones de vuelo desacopladas. Se encontraron los modelos suficientemente representativos de la dinámica para el pitch y yaw, base para el planteamiento de controles por técnicas lineales.

Dos técnicas de control fueron trabajadas. Una basada en controles PID y otra en controles difusos. Ambas presentaron resultados apropiados a nivel de simulación y pruebas sobre la estructura real. Las gráficas producto de las simulaciones y las adquiridas a partir del sistema real soportan tiempos similares. Incluso, se infiere la validez que tiene el modelo teórico del sistema, pues permite llegar a resultados que se ajustan a la respuesta real del prototipo.

Se realizó la instrumentación del sistema físico, lo que permitió la integración de los controles. Para esto, se hizo la incursión en la teoría de "Software In the Loop" y "Hardware In the Loop". Se probaron los controles diseñados, y se mistró la necesidad de realizar una sintonización fina en cada uno de los casos de las técnicas de control abordadas.

Un valor agregado al presente trabajo se puede reflejar en el costo de fabricación y puesta en funcionamiento del sistema. Si se compara con los prototipos que se consiguen en el mercado, este prototipo abarata costos y facilita el acceso a ellos, lo que lo convierte en un elemento de apoyo a la academia. Su fabricación es relativamente simple y la instrumentación se soporta en acelerómetros, que mejoran la precisión pero con consumo de procesamiento adicional para la determinación de ángulos, tema que no se aborda en la presente investigación.

\section{REFERENCIAS}

[1] Vehículo Aéreo no Tripulado. (2008). Recuperado en noviembre de 2010, de http://airvoila.com/vehiculo-aereo-notripulado-uav.

[2] Quanser Inc. (2010). DOF Helicopter. Recuperado en octubre de 2010, de http://www.quanser.com/english/ html/products/fs_product_challenge. asp?lang _ code =english\&pcat _ code $=$ exp-spe\&prod_code $=S 2-$ 2dofheli\&tmpl=1

[3] Universita' Degli Studi di Siena, Dipartimento di Ingegneria dell'Informazione e Scienze Matematiche. (2010), CE150 Helicopter Model. Recuperado en octubre de 2010, 
de http://www.dii.unisi.it/ control/cmr/ altro/heli_ce150_manual.pdf

[4] Fang, Z., Wu, J. \& Li, P. (2008), Control system design and flight testing for a miniature unmanned helicopter. WCICA 2008 7th World Congress, pp. 23152319, 25-27

[5] Dutka A.S., Ordys, A.W. \& Grimble, M.J. (2003). Non-linear predictive control of 2 DOF helicopter model. Proceedings 42nd IEEE Conference, 4, pp. 3954- 3959, 9-12

[6] Kim, B, Chang, Y., Keh, J., Ha, H. \& Lee, M. (2004). Design of 6-DOF attitude controller of hovering model helicopter. IECON 2004. 30th Annual Conference of IEEE, 1, pp. 104- 110, 2-6

[7] Morata Palacios, F. (2009). Controlador Fuzzy de un Quadrotor (Tesis de Maestría inédita). Universidad Complutense de Madrid, Madrid, España.

[8] Londoño, O.M. \& Vélez, C.M. (2009). Identificación del modelo de un mini helicóptero robot usando algoritmos genéticos. VIII Congreso de la Asociación Colombiana de Automática (ACA).

[9] Ruiz Soto, A. (2009), Construcción y Control de una planta didáctica de 2 grados de libertad (Tesis de Maestría inédita). Universidad de Veracruz, Veracruz, México.

[10] Ogata, K. (2003). System Dynamics (4th Ed.). Lugar: Upper Saddle River, New Jersey 07458. Prentice Hall, p. 784.
[11] Ogata, K. (2009). Modern control engineering 5th edition. Lugar: Upper Saddle River, New Jersey 07458. .Prentice Hall, p. 912.

[12] Passino, K. \& Yurkovich, S. (1998). Fuzzy Control. Lugar: 2725 Sand Hill Road, Menlo Park, California 94025. Addison Wesley Longman Inc., p. 522. 